\title{
Role of geomechanically grown fractures on dispersive transport in heterogeneous geological formations
}

\author{
H. M. Nick* \\ Department of Earth Sciences, University of Utrecht, Netherlands \\ A. Paluszny and M. J. Blunt \\ Department of Earth Science and Engineering, Imperial College London, United Kingdom \\ S. K. Matthai \\ Department of Mineral Resources and Petroleum Engineering, Montan University, Austria
}

(Received 22 May 2011; published 4 November 2011)

\begin{abstract}
A second order in space accurate implicit scheme for time-dependent advection-dispersion equations and a discrete fracture propagation model are employed to model solute transport in porous media. We study the impact of the fractures on mass transport and dispersion. To model flow and transport, pressure and transport equations are integrated using a finite-element, node-centered finite-volume approach. Fracture geometries are incrementally developed from a random distributions of material flaws using an adoptive geomechanical finite-element model that also produces fracture aperture distributions. This quasistatic propagation assumes a linear elastic rock matrix, and crack propagation is governed by a subcritical crack growth failure criterion. Fracture propagation, intersection, and closure are handled geometrically. The flow and transport simulations are separately conducted for a range of fracture densities that are generated by the geomechanical finite-element model. These computations show that the most influential parameters for solute transport in fractured porous media are as follows: fracture density and fracture-matrix flux ratio that is influenced by matrix permeability. Using an equivalent fracture aperture size, computed on the basis of equivalent permeability of the system, we also obtain an acceptable prediction of the macrodispersion of poorly interconnected fracture networks. The results hold for fractures at relatively low density.
\end{abstract}

DOI: 10.1103/PhysRevE.84.056301

PACS number(s): 47.56.+r, 47.11.-j

\section{INTRODUCTION}

Understanding the effect of rock fractures on solute transport is a challenging problem for engineers: petroleum engineers characterize and predict the effect of such systems on oil recovery (see, e.g., [1]); hydrogeologists investigate these effects in remediation of contaminated groundwater (see, e.g., [2]), and there is an additional interest in this problem in the context of underground waste disposal, as fractures may facilitate leakage of radionucleids by providing fast flow pathways [3]. Such studies require knowledge of the spatial distribution of rock fractures and their aperture sizes (see, e.g., [4]).

Recent numerical studies show that fracture patterns in layered rocks can be realistically recreated by approximating mechanical behavior in two-dimensional simulations [5-9]. Interest in simulating fracture growth extends across a variety of application fields, including hydraulic fracturing (see, e.g., [10]), structural analysis for civil engineering (see, e.g., [11]), composite material design for aeronautics (see, e.g., [12]), nuclear waste disposal risk assessment (see, e.g., [13]), and analysis of flow and mechanical properties of fractured reservoirs (cf. [14]).

Geomechanical discrete fracture simulation is an alternative to stochastic fracture pattern generation, which provides a means to creating datasets of interacting fractures at different

\footnotetext{
*Present address: Also at Department of Earth Science and Engineering, Imperial College London, United Kingdom; h.m.nick@uu.nl
}

growth stages (see, e.g., [15]). In most stochastic models, density is controlled by the amount, length, and spacing of randomly distributed flaws [16], while in geomechanical models it is a by-product of growth and coalescence. Additionally, randomly generated fractures do not reflect the complex mechanical interactions between fractures that occur during growth, giving rise to their spatial self-organization [9]. Furthermore, stochastic models do not allow for fracture curving. Thus, a fracture is always planar and its orientation predefines its connectivity. In mechanically informed simulations, this is not the case: fractures can grow in many shapes and patterns, and curving and coalescence can greatly enhance connectivity without significantly increasing density [17]. Therefore, while the stochastic method may reproduce the statistics of fracture data measured in the field, it does not capture the more subtle aspects of natural fracture distributions that depend on the rock deformation history and its material properties. In contrast, a geomechanics growth simulator can generate datasets of differing densities corresponding to advancing stages of growth, without changing the underlying spacing and topology of the fracture pattern. Thus, it allows us to study density changes on the same mutating fracture dataset.

Related work on fracture pattern generation relies on a subgrid representation of fractures and on the extension of the finite-element method (FEM) to capture them as discontinuities in the displacement field [5]. The advantage of the finite-element-based modeling of deformation is the simplicity of the numerical discretization of the solved equations. The idea is that by retaining an accurate representation 
of topology and material interfaces, the numerical method is relieved of a submesh representation of the geometry and there is more room to capture complex behavior, such as compaction, damage, and inelastic deformation. An example that couples flow and deformation is the hydraulically driven fracture propagation simulated by Boone and Ingraffea [10] and Secchi et al. [18]. Using an approach where fracture and matrix domains are discretely represented allows for its swift integration into compatible flow codes to measure multiphase flow and other transport properties.

Describing solute transport in terms of average equations is a challenge. Flow velocity variations are responsible for the dispersive movement of contaminants and tracers in heterogeneous porous media (see, e.g., [19]). Berkowitz and Scher [20,21], for example, found anomalous or nonFickian transport by using particle tracking simulations on a set of randomly generated fractures. Many laboratory and field measurements of solute transport in fractured rocks have shown the same behavior [22-25]. Unlike the classical $\mathrm{S}$-shaped solute breakthrough curves of homogeneous porous media in fractured porous media [26,27], these curves are characterized by early breakthrough, long tails, and multiple peaks. Thus, they cannot be captured by the standard advection diffusion equation (ADE). Consequently, there has been significant research on flow and transport in dense and wellinterconnected fracture networks, but little attention has been given to understanding the effect of sparse fracture sets on solute transport.

In this paper, we demonstrate how geomechanically realistic fractures impact solute transport. We achieve this by combining a geomechanic model with a single-phase flow and transport model [28]. The first provides geomechanically grown fracture sets including fracture aperture distributions. The second simulates flow and transport for different stages of fracture growth and densities. We also compute the equivalent aperture size of the analyzed fracture patterns and the equivalent permeability of the system, and aim to forecast solute transport.

This paper is organized as follows. Section II describes the mechanical, flow, and transport simulation, including the setup of the numerical model. Section III presents the results, including the following: (1) a discussion of computational errors in terms of discretization error and convergence of the averaging procedure, (2) a study of the effect of different fracture growth stages on the dispersive behavior, (3) a study of the effect of rock matrix permeability on transport, and (4) a comparison of results obtained from networks with variable and constant aperture.

\section{METHODOLOGY}

This section first presents a brief review of the finiteelement-based deformation kernel, and includes a description of the failure and propagation criteria and a summary of the assumptions of the method. Then we give an overview of the equations for flow and transport in porous media. This is followed by a description of the numerical schemes for flow and transport as well as the setup of the numerical model.

\section{A. Mechanical model}

In linear elastic deformation, stress is related to strain by [29]

$$
\sigma=D_{e}\left(\varepsilon-\varepsilon_{0}\right)+\sigma_{0},
$$

where $\boldsymbol{\varepsilon}=\left\{\varepsilon_{x x}, \varepsilon_{y y}, \varepsilon_{x y}\right\}^{T}$ [-] is the strain vector, $\boldsymbol{\sigma}=$ $\left\{\sigma_{x x}, \sigma_{y y}, \sigma_{x y}\right\}^{T}\left[\mathrm{ML}^{-1} \mathrm{~T}^{-2}\right]$ is the stress vector, and $\sigma_{\mathbf{0}}$ and $\boldsymbol{\varepsilon}_{\mathbf{0}}$ are the initial stress and strain vectors, respectively. The tensor $\boldsymbol{D}_{\boldsymbol{e}}$ is the material stiffness matrix. Assuming plane strain, $\boldsymbol{D}_{\boldsymbol{e}}$ is

$$
\boldsymbol{D}_{e}=\frac{E}{(1+v)(1-2 v)}\left[\begin{array}{ccc}
1-v & v & 0 \\
v & 1-v & 0 \\
0 & 0 & 1-2 v
\end{array}\right],
$$

where $E\left[\mathrm{ML}^{-1} \mathrm{~T}^{-2}\right]$ is Young's modulus and $v[-]$ is Poisson's ratio. At force equilibrium, $\partial \boldsymbol{\sigma}+\boldsymbol{F}=0$, where $\boldsymbol{F}$ is forces and $\partial$ is the kinematic operator as

$$
\boldsymbol{\partial}=\left[\begin{array}{cc}
\frac{\partial}{\partial x} & 0 \\
0 & \frac{\partial}{\partial y} \\
\frac{\partial}{\partial y} & \frac{\partial}{\partial x}
\end{array}\right]
$$

We have implemented a quadratic finite-element solution for the deformation of arbitrarily shaped objects with properties described by Eq. (1).

Modeling multiple crack growth relies on three locally defined criteria: failure, propagation magnitude, and angle [17]. A failure criterion determines if the fracture tip will advance. A subcritical crack growth failure criterion, used herein, prescribes that tips will propagate due to inherent fatigue and corrosive processes that progressively weaken the rock tips [30]. Propagation speed is related to fracture length. Within a group of quasistatically propagating cracks, it is described by a Paris-like power law [31-33]. We measure the elastic strain energy release rate, $G$, at each tip and plain strain as $G=\left(1-v^{2}\right) / K_{I}^{2} E$ and for plane stress as $G=K_{I}^{2} / E$, where $K_{I}\left[\mathrm{ML}^{-3} \mathrm{~T}^{-2}\right]$ is the fraction of the stress intensity factor corresponding to mode $I$. Additionally, we monitor the tip with the maximum energy concentration $G_{\max }=\|G\|_{\infty}$, the $L$-infinity norm of $G$, assuming that it is the fastest propagating tip.

To compute the distance a crack tip will advance at any propagation step we use the well-established Paris-type propagation criterion originally defined by Charles [34] and extended by Renshaw and Pollard [9]. This criterion relates the energy accumulated around a specific tip with the maximum energy of all tips and weight growth with an empirical velocity index, $\alpha$. Thus, tips with the highest $G$ advance faster than the rest. It follows that [9]

$$
l_{\mathrm{adv}}=l_{\max }\left(\frac{G}{G_{\max }}\right)^{\alpha=0.35},
$$

where $l_{\text {adv }}$ is the propagation length and $l_{\max }$ is the maximum length increase at any propagation step. We use a fixed velocity exponent of 0.35 identified by exhaustive experimentation to yield realistic fracture patterns in rock analogs [9]. Finally, the fracture propagation angle is computed for each individual fracture from the local maximum circumferential stress at the tip [35]. 
Initially, any model is populated with a set of randomly distributed and oriented flaws. Flaws grow into fractures represented by two-dimensional closed polygons when the model is deformed. At each loading step the mesh is adapted to capture the emerging fracture geometry. This is accomplished by a high-level Picard iteration allowing fractures to advance until there is insufficient strain to induce further propagation. Further details of the method, including its validation, can be found in Paluszny and Matthäi $[15,17]$.

The assumptions of this mechanical model are as follows: (1) the original material is brittle, homogeneous, isotropic, and linearly elastic; (2) only tensile failure is considered; (3) fracture tips are initially pointy, and linearly approximated by an elliptical shape; (4) propagation is quasistatic and strain rate independent; (5) there is no cohesion or traction between fracture walls; and, (6) deformation is simulated in two dimensions in plane strain conditions.

\section{B. Flow and transport modeling}

The specific discharge $\mathbf{u}\left[\mathrm{LT}^{-1}\right]$, in Darcy's law,

$$
\mathbf{u}=-\frac{\mathbf{k}}{\mu} \nabla P,
$$

is a function of $\mathbf{k}$ the intrinsic permeability tensor $\left[\mathrm{L}^{2}\right], \mu$ the dynamic viscosity $\left[\mathrm{ML}^{-1} \mathrm{~T}^{-1}\right]$, and $\nabla P$ the pressure gradient $\left[\mathrm{ML}^{-1} \mathrm{~T}^{-2}\right]$. Conservation of mass is given by the continuity equation

$$
\nabla \cdot\left(\frac{\mathbf{k}}{\mu} \nabla P\right)=0 .
$$

The mass balance for a nonreactive and nonadsorbing solute in a nondeformable porous medium is given by

$$
\phi \frac{\partial c}{\partial t}+\nabla \cdot\left(\mathbf{u} c-D_{\text {eff }} \nabla \mathbf{c}\right)=0
$$

where $c$ denotes the concentration $\left[\mathrm{ML}^{-3}\right], t$ represents time $[T], D_{\text {eff }}$ is the effective molecular diffusion $\left[\mathrm{L}^{-2} \mathrm{~T}^{-1}\right]$, and $\phi$ is porosity.

\section{Second-order implicit scheme}

We solve Eqs. (6) and (7) implicitly using the operator splitting method [28]. First we obtain the pressure field by solving the pressure equation on a linear finite-element discretization. Then the transport equation is solved using a conservative finite-volume method (FVM) discretized on a virtual finite-volume mesh constructed around the nodes of the finite-element mesh (Fig. 7 of Ref. [36]). This method provides a mass conservative transport scheme. The fluid pressure is solved for steady state by solving the integration of Eq. (6) over the domain $\Omega \subset \mathbb{R}^{d}(d=2,3)$ for $\mathbf{x} \in \Omega$ :

$$
\int_{\Omega} \nabla \mathbf{N}^{T} \frac{\mathbf{k}}{\mu} \nabla N d \mathbf{x}=0
$$

The superscript $T$ refers to the transpose of the element interpolation function vector or matrix of spatial derivatives and $\mathbf{N}$ and $\nabla \mathbf{N}$ represent its interpolation function vector and matrix of spatial derivatives obtained from each element, respectively. The transport equation is solved using a fractional step method in which the diffusion term of the ADE is discretized using an implicit FEM and the advection term of Eq. (7) is discretized using an implicit FVM. By analogy with the pressure equation, finite-element integration of the diffusion term in Eq. (7) over the domain $\Omega$ yields

$$
\begin{aligned}
& \left(\int_{\Omega} \mathbf{N}^{T} \phi \mathbf{N} d \mathbf{x}-\Delta t \int_{\Omega} \nabla \mathbf{N}^{T} D_{\mathrm{eff}} \nabla \mathbf{N} d \mathbf{x}\right) c^{t+\Delta t} \\
& \quad=\left(\int_{\Omega} \mathbf{N}^{T} \phi \mathbf{N} d \mathbf{x}\right) c^{t} .
\end{aligned}
$$

For the advection term in Eq. (7), using piecewise constant $F V$ interpolation functions, $M_{j}$ for each finite-volume $j$ and a first-order upwind scheme, volume integration $V$, gives

$$
\phi \int_{V} \mathbf{M} c^{t+\Delta t} \mathbf{d} V+\Delta t \oint_{V}(\mathbf{n} \cdot \mathbf{u}) c^{t+\Delta t} d S=\phi \int_{V} \mathbf{M} c^{t} \mathbf{d} V
$$

where $\mathbf{n}$ represents the normal vector to the outward-facing surface element. To make the transport scheme second-order accurate in space, we calculate estimates of the gradient of the transported variable for each control volume facet. Then we apply the minmod slope limiter to suppress spurious oscillations that occur when the gradient of the transported variable is overestimated. This guarantees that the transport scheme becomes total variation diminishing (TVD) [28].

The hybrid finite element-finite volume method is implemented in Complex Systems Platform (CSMP++) [36-38] and is capable of considering fractures represented by lower-dimensional elements. This approach, however, is only applicable when the fractures have a higher permeability than the rock matrix. We use the system algebraic multigrid method (SAMG) to solve the ensuing FEM linear algebraic equations [39].

\section{Fracture aperture}

For the flow simulation, the rock matrix is discretized by triangular elements while fractures are represented by line elements, corresponding to their centerlines. To retain the fracture aperture we store a scalar value at each node in the variable $a_{f}$ along each fracture:

$$
a_{f}=\left\langle a_{f o}|\cdots| a_{f i}|\cdots| a_{f n}\right\rangle,
$$

where fracture $f$ has $n$ nodes along its centerline. Due to the lower dimensional representation of the fracture, we weight properties defined at the lines, such as permeability and porosity, with the local aperture to capture the actual thickness of the reduced element [40]. Figure 1 illustrates the piecewise mapping of the apertures along the fracture onto its centerline.

One could also use constant aperture values for all fractures. Two constant values for fracture aperture in an entire model appear meaningful: the weighted segment length average $\left(a_{\text {avg }}\right)$ and equivalent $\left(a_{\text {equiv }}\right)$. The weighted average aperture is calculated

$$
a_{\mathrm{avg}}=\frac{\sum_{i}^{n} a_{f i} l_{i}}{\sum_{i}^{n} l_{i}}
$$

where $l_{i}$ is the length of fracture element $i \in[1, n]$. To calculate the equivalent aperture size, we calculate the equivalent 


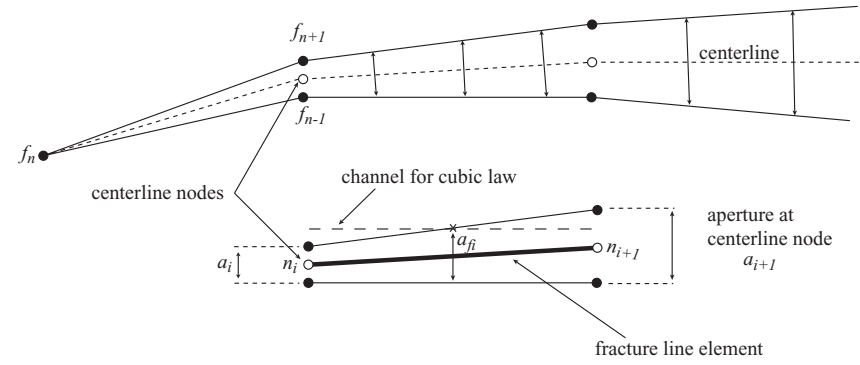

FIG. 1. Fracture centerline and aperture measurement. Black dots are nodes on the fracture wall (i.e., $f_{n}, f_{n+1}$, and $f_{n-1}$ ). White dots are the corresponding centerline nodes, $n_{i}$ and $n_{i+1} . f_{n}$ is a fracture tip. $a_{i}$ is the aperture of the fracture at $n_{i}$. The thick line represents a fracture line element on the centerline. The aperture assigned to the local line element, $a_{f i}$, is the average between $a_{i}$ and $a_{i+1}$.

permeability of the model [41] using the mechanical simulation results, i.e., considering fractures with variable aperture sizes. Then, by using an iterative method we determine a single valued equivalent aperture ( $\left.a_{\text {equiv }}\right)$ that yields the same equivalent permeability.

\section{Analysis strategy}

We use the output of the mechanical simulations as input for the transport simulations. For selected iteration levels and fracture densities we output the geometry and aperture sizes to run the transport simulations. These are conducted with the second-order implicit TVD method.

In the mechanical simulations we have employed a $4 \times$ $1 \mathrm{~m}$ model with 100 initial flaws. Their position is random following a uniform probability distribution, and their size obeys a Gaussian distribution with a mean of $0.004 \mathrm{~m}$ and standard deviation $0.045 \mathrm{~m}$. All flaws are initially horizontal and have a minimum spacing of $1.2 \mathrm{~cm}$. We assume rock properties that could typically be a limestone: Young's modulus of $20 \mathrm{GPa}$, Poisson's ratio of 0.25 , and subcritical toughness $K_{I C}$ of $1.5 \mathrm{MPa} \mathrm{m}^{1 / 2}$. We fix the lower position of model boundary and apply an extensional boundary normal displacement of $10^{-3} \mathrm{~m}$ at the top.

Flow and transport simulation are performed on a subregion of the mechanical model, with a size of $2 \times 1 \mathrm{~m}$. For this purpose, a tracer is uniformly distributed, $c=1 \mathrm{~kg} / \mathrm{m}^{3}$, in a 1-cm-thick vertical slit along the left side of the model. Elsewhere, concentration is set to zero. Tracer-free fluid is then injected through the left boundary inducing a 1 $\mathrm{MPa} / \mathrm{m}$ pressure gradient that is held constant throughout the simulation. Note that there is no density or viscosity variation in this study. These conditions apply to all of our numerical experiments. Matrix permeability for most cases is set to $10 \mathrm{mD}$ while matrix porosity is $30 \%$. We assume that all fractures are open and calculate fracture permeability from local aperture using the parallel plate law $\left(k_{f}=a_{f}^{2} / 12\right)$. This assumes that the flow is laminar and the fracture has smooth walls with a local separation of $a_{f}[42,43]$. Fracture permeability, $k_{f}$, is defined as piecewise constant along the line elements.

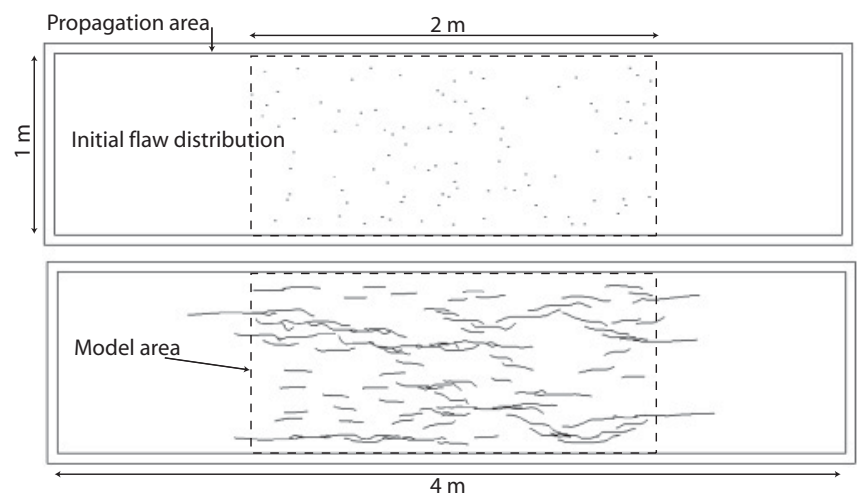

FIG. 2. An initial flaw distribution and a developed fracture set of a growth simulation. Flaws are grown within the propagation area; for flow simulations a model area is extracted from the original dataset.

\section{RESULTS AND DISCUSSION}

\section{A. Fracture patterns}

In all mechanical models, initially, flaws grow following straight paths. Once they become larger, they start to mechanically interact with proximal fractures by influencing the stress field around their tips (Fig. 2). Thus, connectivity does not increase smoothly as a function of density; instead, it increases in a steep stepwise manner. The fracture geometry and aperture distribution of a model realization at three stages of growth are shown in Fig. 3.

The initial distribution of the flaws plays an important role in determining the final pattern, because it determines where fractures start to propagate. However, their orientation exhibits little effect on the final network. In contrast, spacing has a quantitative impact on the final pattern. Areas without flaws act as "stronger" areas of the model, and in turn exhibit less fracturing. A thorough discussion of the generated fracture pattern is presented in Paluszny and Matthäi [15].

\section{B. Discretization error}

The mechanical model uses dynamic adoptive mesh refinement; see Paluszny and Matthäi [15]. In order to select an adequate mesh size for the flow and transport simulations, we conduct calculations on five triangular element meshes (Meshes 1-5), where Mesh 1 is the coarsest and Mesh 5 is the finest (Table I). Using a finer element size either uniformly or in a spatially adoptive fashion are possible ways to increase the quality of the solution. The numerical solution of these simulations on one realization shows convergence by increasing the level of refinement. The $L^{2}$ error norms, $\| c_{j}-$ $c_{r} \|=\sqrt{\sum_{i=1}^{i=N_{i}}\left(c_{j}^{i}-c_{r}^{i}\right)^{2}}$, for different mesh refinements, $j$, are calculated by taking the solution of Mesh 5 as the reference solution, $c_{r}$. Here, $c_{j}^{i}$ denotes the output concentration at different time, and $N_{i}$ is the number of time steps. These errors and the error reduction, $\left\|c_{j}-c_{j+1}\right\|$, are presented in Table I. Considering computational time and reduced errors measured for Mesh 4, we choose a mesh size of $1 \mathrm{~cm}$ for the rock matrix and $0.25 \mathrm{~cm}$ for the fractures. The results show that regions requiring more refinement are regions with the highest velocity gradients. 

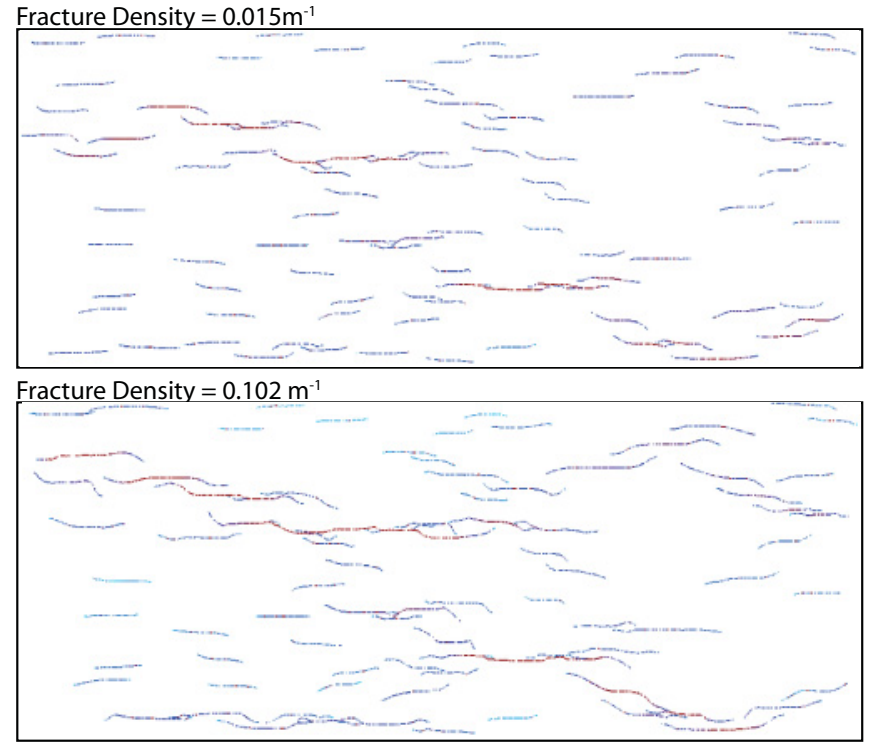

Fracture Density $=0.230 \mathrm{~m}^{-1}$

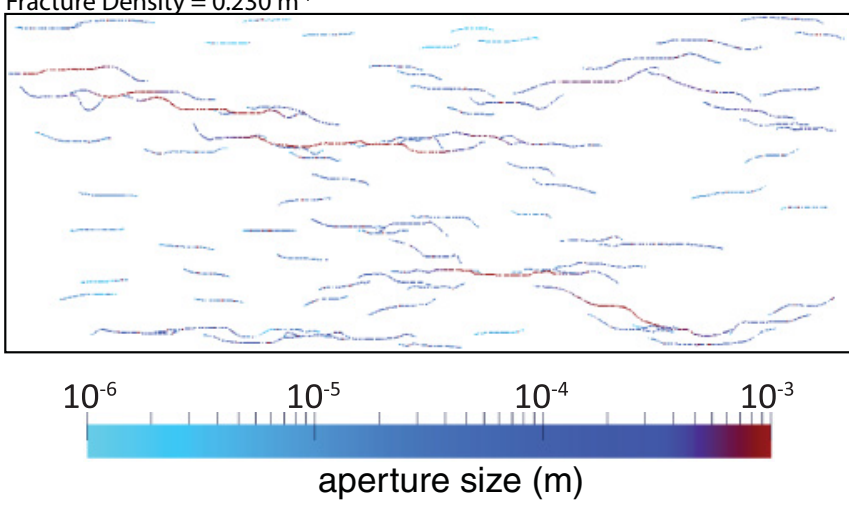

FIG. 3. (Color online) Mechanically grown fracture networks at different growth stages (after 20, 50, and 80 iterations) with a maximum aperture size of $0.001 \mathrm{~m}$. Colors are indicative of fracture aperture.

\section{Ensemble breakthrough curves}

We take the ensemble average of the breakthrough curves, at the right boundary, over $n$ realizations. We use the criterion

$$
E^{i}=\left\|\langle c\rangle^{i}-\langle c\rangle^{i-1}\right\|
$$

to determine the number of realizations needed for averaging. $E^{i}$ shows the convergence of the ensemble breakthrough curves (Fig. 4). Here \langle\rangle$^{i}$ denotes the breakthrough average

TABLE I. Discretization errors as a function of mesh refinement: $e_{m}$ are matrix element sizes, $e_{f}$ are fracture element sizes. Meshes 1 , 3 , and 5 are uniformly refined everywhere, whereas Meshes 2 and 4 are more refined at the fractures. Mesh 1 is the coarsest while Mesh 5 is the finest.

\begin{tabular}{lccccc}
\hline \hline Mesh & $e_{m}(\mathrm{~cm})$ & $e_{f}(\mathrm{~cm})$ & Nodes & $\left\|c_{j}-c_{j+1}\right\|$ & $\left\|c_{j}-c_{r}\right\|$ \\
\hline Mesh 1 & 1 & 1 & 18470 & $1.9 \times 10^{-2}$ & $1.5 \times 10^{-2}$ \\
Mesh 2 & 1 & 0.5 & 29679 & $9.3 \times 10^{-3}$ & $1.0 \times 10^{-2}$ \\
Mesh 3 & 0.5 & 0.5 & 73881 & $9.9 \times 10^{-3}$ & $9.6 \times 10^{-3}$ \\
Mesh 4 & 1 & 0.25 & 52917 & $6.6 \times 10^{-3}$ & $6.6 \times 10^{-3}$ \\
Mesh 5 & 0.25 & 0.25 & 291242 & - & - \\
\hline \hline
\end{tabular}

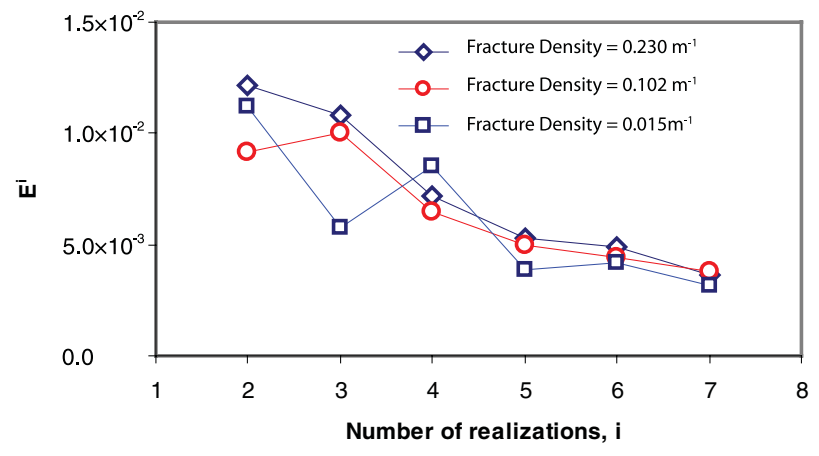

FIG. 4. (Color online) Average convergence error for different growth stages. $E^{i}$ is $\left\|\langle c\rangle^{i}-\langle c\rangle^{i-1}\right\|$ and \langle\rangle$^{i}$ denotes the breakthrough average of $i$ realizations.
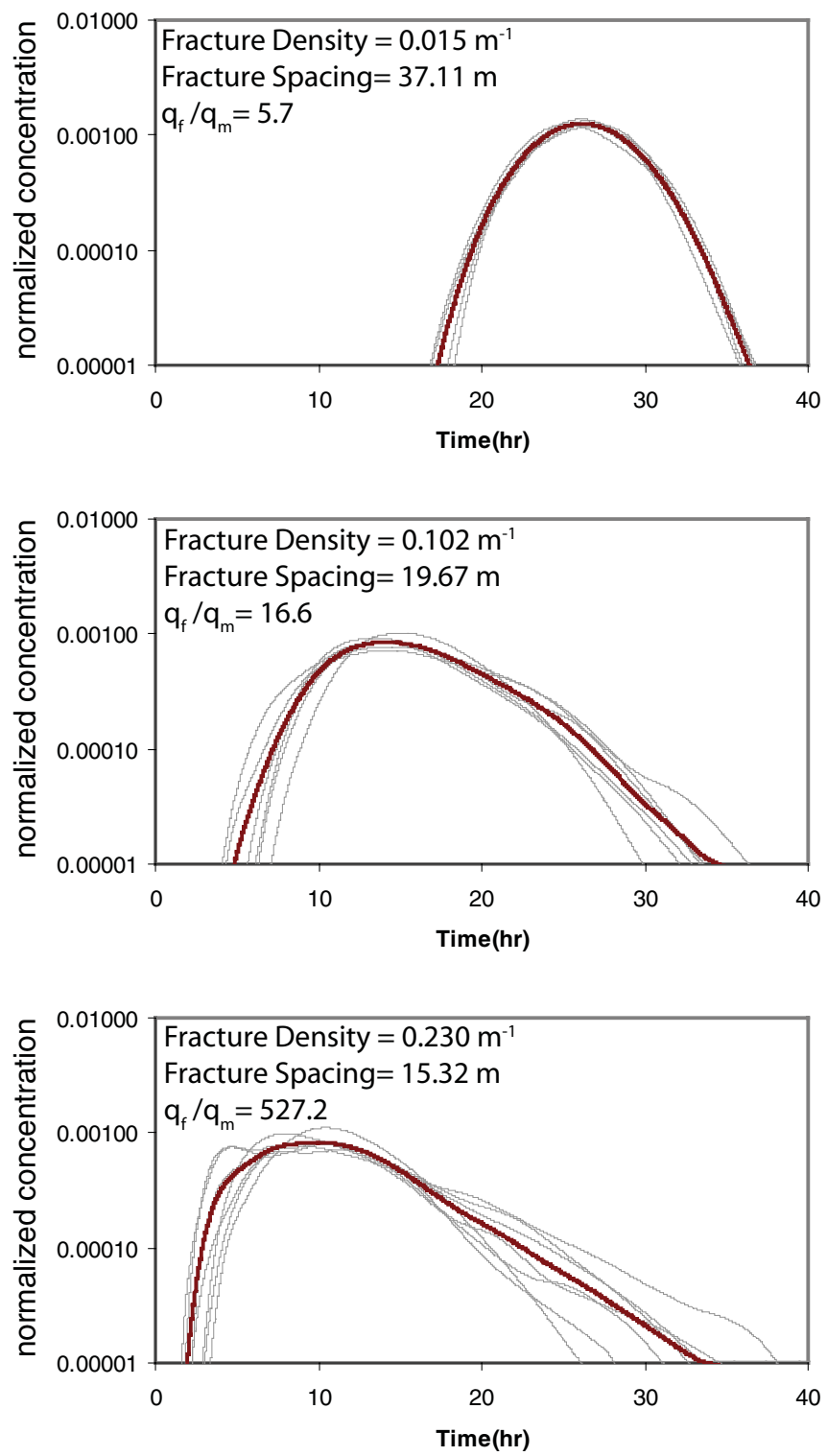

FIG. 5. (Color online) Breakthrough curves for the model with different realizations at different fracture network growth stages. Bold lines indicate the average, and faint lines indicate the breakthrough curves for each realization. 


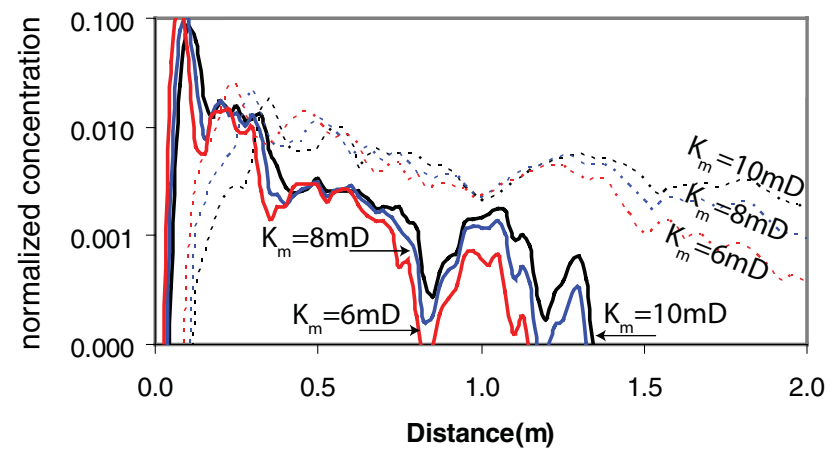

FIG. 6. (Color online) Concentration profiles for a single fracture geometry realization and different matrix permeabilities at two different times. Bold lines are the profiles after 1 hour and the dashed lines are the concentration profiles after 5 hours.

of $i$ realizations. The variation of the breakthrough curves for each realization around a mean value is higher for more developed fracture sets. The averaged breakthrough curves are presented in Fig. 5. We also calculate the fracture-matrix flux ratio, $q_{f} / q_{m}$, as stressed by Matthäi and Belayneh [44] the importance of this ratio on flow pattern in fractured rock.

\section{Effect of rock matrix permeability}

Fracture patterns in this study are not well interconnected, even in later stages of growth (see Fig. 3). Therefore, flow through the matrix has a major effect on total flow and transport. Matthäi and Belayneh [44], who employed field fracture set to address flow partitioning between fractures and a permeable rock matrix, found that the rock matrix flow is negligible and the fracture flow becomes dominant if the fracture matrix permeability ratio is greater than $10^{6}$. We conduct simulations for seven realizations with permeabilities ranging from 0.01 to $10 \mathrm{mD}$. Model properties, including fracture density and fracture spacing, are fixed, allowing the fracture-matrix flux ratio to vary. Figure 6 compares concentration profiles of one realization with different rock permeabilities after 1 hour and 5 hours. The plume transverses slower in the model with lower matrix permeability. This slow

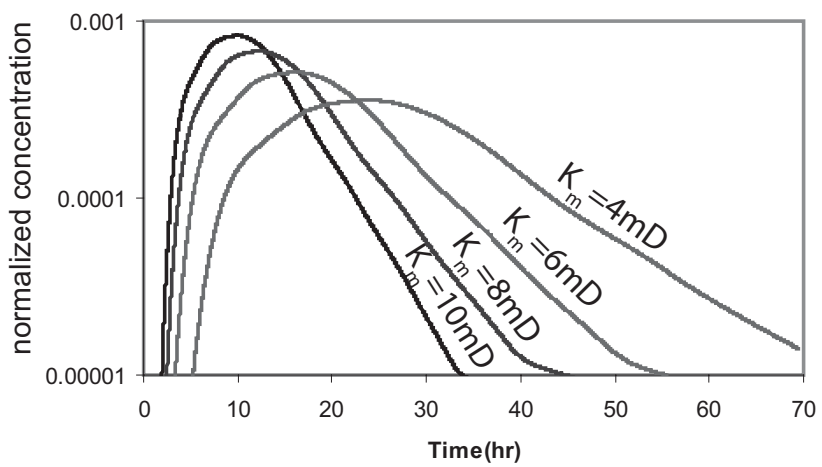

FIG. 7. Breakthrough curves for the model with different matrix permeabilities. Four different matrix permeabilities are compared: $k_{m}=10,8,6,4 \mathrm{mD}$.

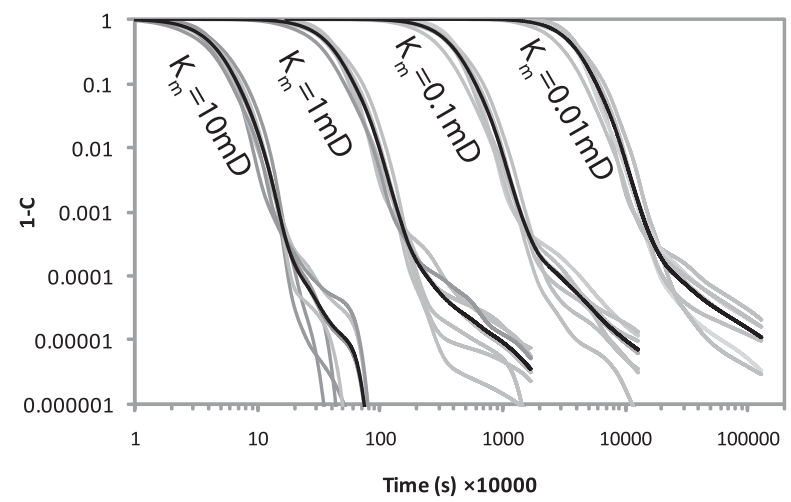

FIG. 8. Breakthrough curves for seven realizations and a wider range of matrix permeability: $k_{m}=0.01,0.1,1,10 \mathrm{mD}$. The bold lines are the average breakthrough curves, and the faint lines indicate the breakthrough curves for each realization.

movement in the matrix and corresponding higher fracturematrix flux ratio leads to more anomalous behavior. For each matrix permeability value the average breakthrough curves of seven realizations are averaged (Fig. 7). It is clear that smaller matrix permeability values cause a stronger localization of flow in fractures. This can also be seen in Figs. 8 and 9 for a wider range of matrix permeability with continuous injection of the tracer through the left boundary. The dispersive behavior of such systems is, however, controlled by matrix permeability as fractures are not well connected. The average flow velocity decreases by increasing the matrix permeability, as the far-field pressure gradient is constant. This suggests that increasing the average velocity reduces the dispersive behavior of the system. The observation of Odling et al. [45] on dispersion in a microfractured granite confirms this, as they concluded that the fluid velocity is considerably influential on the dispersive properties of a fractured rock. They attributed the long tail of breakthrough curves to hydrodynamic retardation, which is significantly altered by flow velocity.

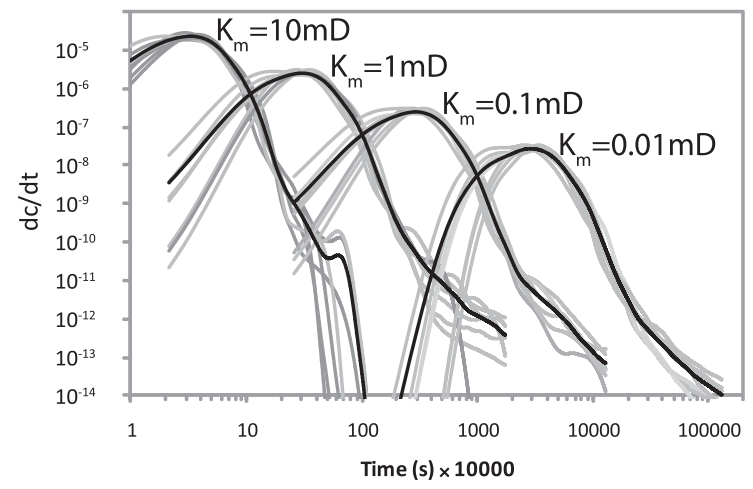

FIG. 9. Time derivative of breakthrough curves for the model with four different matrix permeabilities: $k_{m}=0.01,0.1,1,10 \mathrm{mD}$. The bold lines are the ensemble averages of the seven realizations, and the faint lines indicate breakthrough curves of individual realizations. 

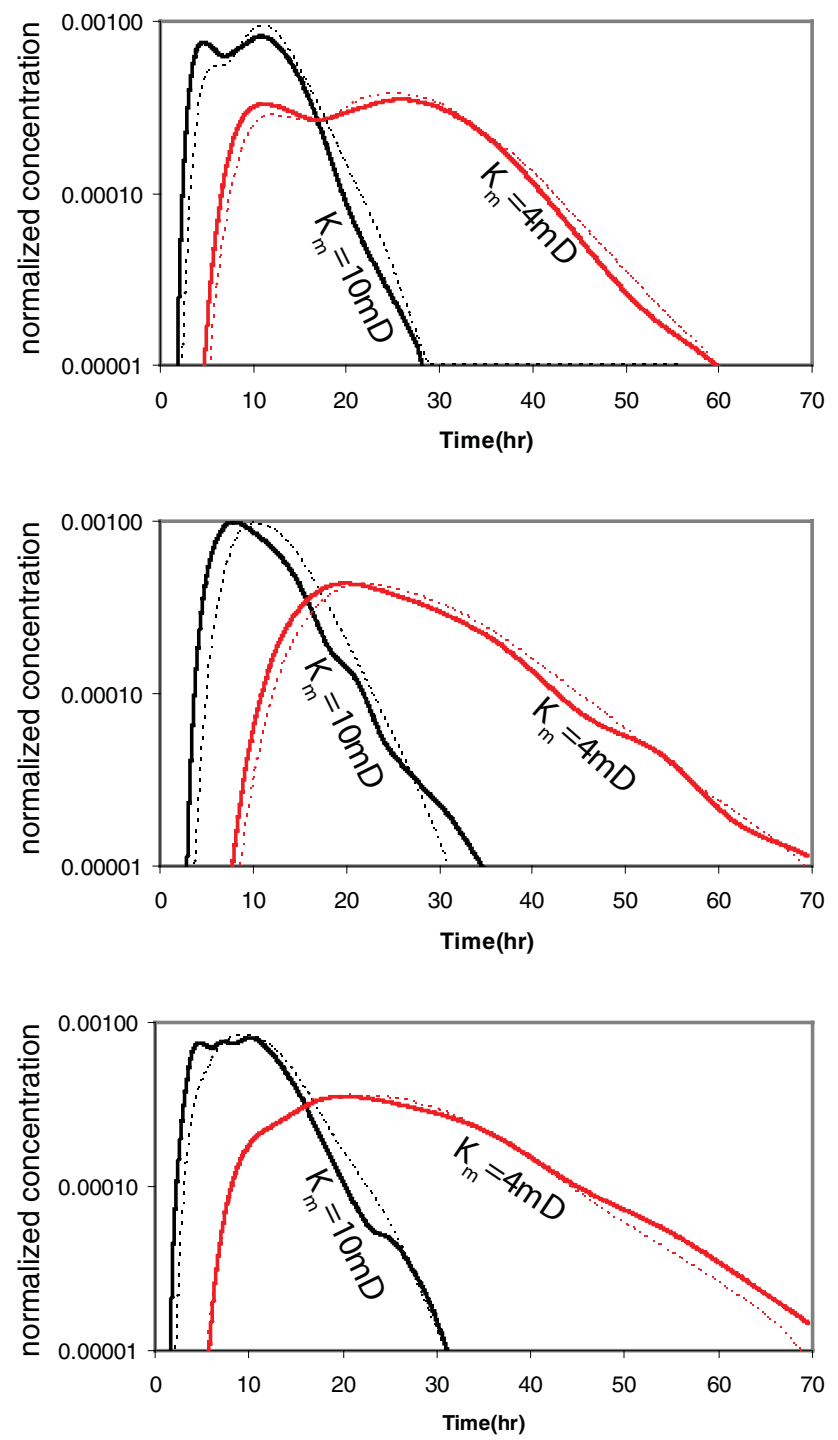

FIG. 10. (Color online) Comparison of the breakthrough curves of three realizations between models with geomechanical aperture distribution (solid lines), and ones with equivalent aperture sizes (dotted lines). Flow simulations for each realization are conducted for two different rock matrix permeabilities: $k_{m}=4,10 \mathrm{mD}$.

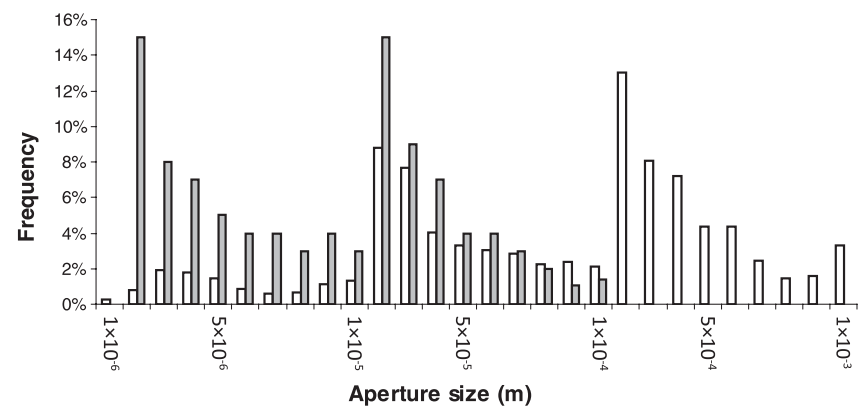

FIG. 11. Aperture size histogram resulting from two different displacements of $0.001 \mathrm{~m}$ (white) and $0.0001 \mathrm{~m}$ (gray), at the model boundaries.
TABLE II. Average and equivalent aperture sizes for different displacements and different rock matrix permeabilities. $D$ is the displacement, $k_{m}$ is the matrix permeability, $a_{\text {avg }}$ is the average aperture, and $a_{\text {equiv }}$ is the equivalent permeability.

\begin{tabular}{lcccc}
\hline \hline$D(\mathrm{~m})$ & 0.001 & 0.001 & 0.0001 & 0.0001 \\
$K_{m}(\mathrm{mD})$ & 10 & 4 & 10 & 4 \\
$a_{\text {avg }}\left(\times 10^{-5} \mathrm{~m}\right)$ & 27.8 & 27.8 & 7.96 & 7.96 \\
$a_{\text {equiv }}\left(\times 10^{-5} \mathrm{~m}\right)$ & 8.66 & 7.88 & 3.98 & 3.90 \\
\hline \hline
\end{tabular}

\section{E. Constant fracture aperture size}

We define equivalent aperture as the single aperture that, when assigned to all fractures of the pattern, yields the same equivalent permeability as using geomechanical apertures. The equivalent aperture has also been used to calculate transport. Here, we compare the transport simulations with the full geomechanical aperture distribution with constant equivalent aperture size on the same fracture dataset. This procedure is repeated for different realizations with two different rock matrix permeabilities (Fig. 10).

Two concentration peaks result from the existence of two main separate pathways [Fig. 10(a)] also appear in the breakthrough curve result of the simplified model. The main difference is the time to breakthrough. It is earlier for the variable aperture model. The slopes of the breakthrough curves are similar.

This result suggests that using equivalent aperture size, computed based on the equivalent permeability of the system, yields acceptable predictions of dispersion in fractured media. Note that further study is required to examine this behavior in well-connected fracture networks and three-dimensional fractured porous media. However, this provides some assurance that the equivalent aperture size does represent adequately flow and transport, particularly, for poorly interconnected fracture networks. The variation in breakthrough curves of different realizations suggests that fracture geometry is more influential on solute transport behavior than aperture size variation. This is in accordance with the findings of Cey et al. [46] for a single fracture. They showed that in a partially saturated medium, aperture variability within individual fractures has a minor effect on the predictability of the flow simulations. Nevertheless, finding the equivalent aperture size in the field

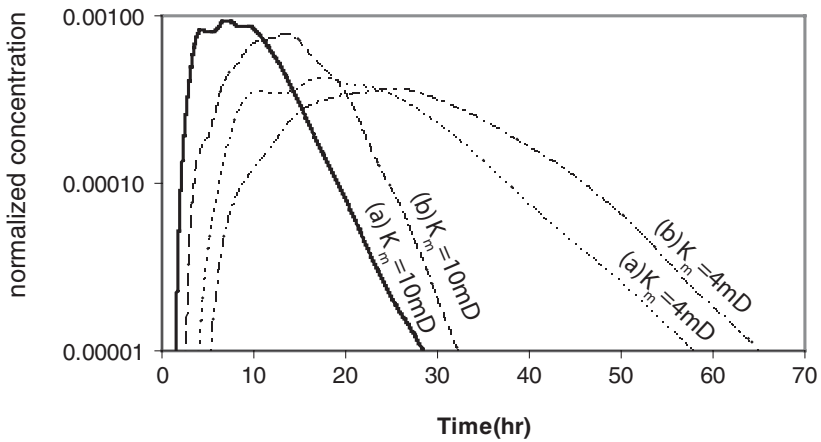

FIG. 12. Breakthrough curves for different displacements of (a) $0.001 \mathrm{~m}$ and (b) $0.0001 \mathrm{~m}$, and different matrix permeabilities: $k_{m}=4$ and $10 \mathrm{mD}$. 

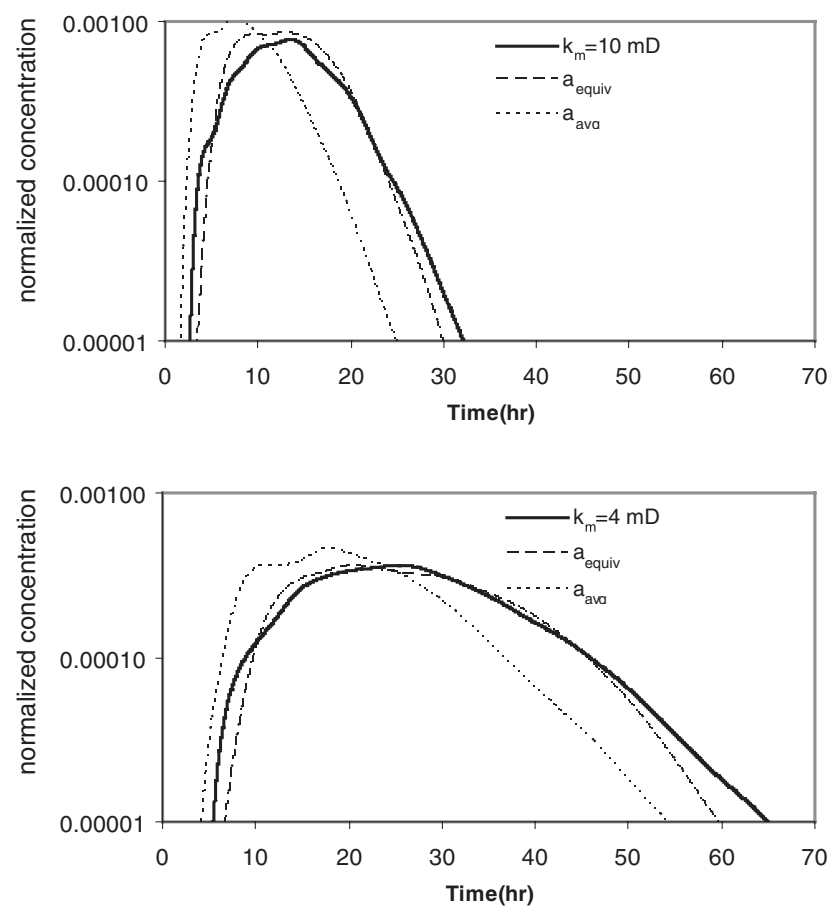

FIG. 13. Breakthrough curves calculated for different aperture distributions: varying aperture (solid lines), equivalent aperture (dashed lines), and weighted average aperture (dotted lines), for a model with two matrix permeabilities, $k_{m}=4,10 \mathrm{mD}$, and a displacement of $1 \mathrm{~mm}$.

is quite challenging; using a constant value for the aperture sizes reduces the complexity of the simulations.

\section{F. Displacement}

We apply two different displacements at the top and bottom of the existing model to examine the effect of the boundary conditions on solute transport. Two different displacements ( $1 \mathrm{~mm}$ and $0.1 \mathrm{~mm}$ ) are set at the model boundaries. The former yields larger aperture sizes and higher heterogeneity. The fracture aperture size histogram for both simulations is plotted in Fig. 11 on logarithmic bins. Transport simulations are presented for two different matrix permeabilities. We calculate equivalent aperture sizes and geometric average aperture sizes. Table II illustrates these value for different scenarios.

Figure 12 illustrates the effect of displacement on the breakthrough curves for two different matrix permeabilities. Larger dilatation, for the boundary condition, results in earlier breakthrough and higher concentration peaks because the equivalent permeability of the model is higher. Figure 13 compares the breakthrough curves predicted by different aperture sizes for the larger displacement of $1 \mathrm{~mm}$. The results for equivalent apertures are in reasonable agreement with those with obtained for varying fracture sizes. The $L^{2}$ error norm is equal to $0.6 \%$ for the model with the matrix permeability of $10 \mathrm{mD}$ and $0.1 \%$ for the matrix permeability of $4 \mathrm{mD}$; whereas using geometric average aperture yields a higher error of $1.8 \%$ and $0.8 \%$, respectively. Paluszny and Matthäi [15] also found that the geometric average aperture does not yield the same equivalent permeability as variable aperture sizes. However, breakthrough curves based on equivalent aperture reveal less a dispersive behavior in comparison with breakthrough curves predicted by using variable aperture.

\section{CONCLUSIONS}

In this work we combine a geomechanical model that produces realistic fracture datasets, including detail aperture distributions, with a flow and transport model. The key findings of this study are as follows:

(i) Our results corroborate earlier findings that naturally fractured media exhibit anomalous transport provided that fractures are developed. The results demonstrate highly dispersed plumes and long tails in the breakthrough curves for fractured media.

(ii) Matrix permeability, fracture density, and the $q_{f} / q_{m}$ ratio show an explicit impact on the dispersive behavior of solute transport in fractured media.

(iii) Using the equivalent aperture size, instead of the actual aperture distribution, reduces detail in breakthrough curves while resembling transport trends for all cases. Our observations suggest that applying the equivalent aperture size calculated based on the equivalent permeability of the system for flow and transport modeling provides a satisfactory result for many applications.

\section{ACKNOWLEDGMENTS}

We thank the sponsors of the ITF project on Improved Simulation of Flow in Fractured and Faulted Reservoirs, and the Technology Strategy Board (TSB), for supporting this research. This work was partially supported by the Center-in-Development Award to Utrecht University (No KUK-C1-017-12) by King Abdullah University of Science and Technology. Dr. Matthew Piggott receives our thanks for scientific feedback and comments. We thank the Imperial College High Performance Computing Service for the use of their cluster.
[1] S. K. Matthäi, A. Mezentsev, and M. Belayneh, SPE Res. Eval. Eng. 10, 740 (2007).

[2] A. A. Keller, M. J. Blunt, and P. V. Roberts, Transp. Porous Media 38, 189 (2004).

[3] H. H. Harrar, L. C. Murdoch, B. Nilsson, and K. E. Klint, Hydrogeol. J. 15, 1473 (2007).
[4] P. H. S. W. Kulatilake, J. Park, P. Balasingam, and R. Morgan, Geotech. Geol. Eng. 26, 269 (2008).

[5] T. Belytschko and T. Black, Int. J. Numer. Meth. Eng. 45, 601 (1999).

[6] R. Huang, N. Sukumar, and J. H. Prevost, Int. J. Solids Struct. 40, 7539 (2003). 
[7] A. R. Ingraffea and V. Saouma, Fracture Mechanics of Concrete, Chapter Numerical Modelling of Discrete Crack Propagation in Reinforced and Plain Concrete (Martinus Nijhof, Leiden, 1985), pp. 171-225.

[8] J. E. Olson, J. Geophys. Res. 98, 12251 (1993).

[9] C. E. Renshaw and D. D. Pollard, J. Geophys. Res. 99, 9359 (1994).

[10] T. J. Boone and A. R. Ingraffea, Int. J. Numer. Anal. Meth. Geomech. 14, 27 (1990).

[11] Z. P. Bazant and M. Verdure, J. Eng. Mech. ASCE 133, 308 (2007).

[12] P. P. Camanho, C. G. Davila, and S. T. Pinho, Fatigue Fract. Eng. Mech. 27, 745 (2006)

[13] B. Shen, O. Stephansson, M. Rinne, H. S. Lee, L. Jing, and K. Rosho, Int. J. Rock Mech. Mining Sci. 41, 472 (2004).

[14] M. Zoback, Reservoir Geomechanics (Cambridge University Press, Cambridge, 2007).

[15] A. Paluszny and S. K. Matthäi, J. Geophys. Res. 115, B02203 (2010).

[16] W. S. Dershowitz and H. H. Einstein, Rock Mech. Rock Eng. 21, 21 (1988).

[17] A. Paluszny and S. K. Matthäi, Int. J. Solids Struct. 46, 3383 (2009).

[18] S. Secchi, L. Simoni, and B. A. Schreer, Int. J. Numer. Anal. Meth. Geomech. 31, 331 (2004).

[19] H. M. Nick, R. Schotting, M. Gutierrez-Neri, and K. Johannsen, Transp. Porous Media 78, 11 (2009).

[20] B. Berkowitz and H. Scher, Water Resour. Res. 31, 1461 (1995).

[21] B. Berkowitz and H. Scher, Phys. Rev. Lett. 79, 4038 (1997).

[22] M. W. Becker and A. M. Shapiro, Water Resour. Res. 36, 1677 (2000).

[23] K. A. Cliffe, D. Gilling, N. L. Jefferies, and T. R. Lineham, J. Contam. Hydrol. 13, 73 (1993).

[24] V. Cvetkovic and H. Cheng, Water Resour. Res. 44, W12447 (2007).
[25] P. Vilks and M. Baik, J. Contam. Hydrol. 47, 197 (2001).

[26] B. Berkowitz, Adv. Water Res. 25, 861 (2002).

[27] S. Geiger, A. Cortis, and J. T. Birkholzer, Water Resour. Res. 46, W12530 (2010).

[28] S. K. Matthäi, H. M. Nick, C. Pain, and I. Neuweiler, Transp. Porous Media 83, 289 (2009).

[29] R. D. Cook, D. S. Malkus, and M. E. Plesha, Concepts and Applications of Finite Element Analysis, 3rd ed. (Wiley, New York, 1989).

[30] B. K. Atkinson, J. Geophys. Res. 89, 4077 (1984).

[31] B. K. Atkinson and P. G. Meredith, Fracture Mechanics of Rock, Chap. 4 (Academic, New York, 1987), pp. 477-526.

[32] D. Broek, Elementary Engineering Fracture Mechanics (Kluwer Academic, Dordrecht, 1986).

[33] M. Kachanov, Int. J. Solids Struct. 23, 23 (1987).

[34] R. J. Charles, J. Appl. Phys. 29, 1549 (1958).

[35] B. Cotterell and J. R. Rice, Int. J. Fract. 16, 155 (1980).

[36] A. Paluszny, S. K. Matthäi, and M. Hohmeyer, Geofluids 7, 186 (2007).

[37] S. Geiger, S. Roberts, S. K. Matthäi, C. Zoppou, and A. Burri, Geofluids 4, 284 (2004).

[38] H. M. Nick and S. K. Matthäi, Vadose Zone J. 10, 299 (2011).

[39] K. Stüben, J. Comput. Appl. Math. 128, 281 (2001).

[40] R. Juanes, J. Samper, and J. Molinero, Int. J. Numer. Methods Eng. 54, 1751 (2002).

[41] S. K. Matthäi and H. M. Nick, AAPG Bull. 93, 1621 (2009).

[42] R. L. Kranz, A. D. Frankel, T. Engelder, and C. H. Scholz, Int. J. Rock Mech. Min. Sci. Geomech. Abstr. 16, 225 (1979).

[43] P. A. Witherspoon, J. S. Y. Wang, K. Iwai, and J. E. Gale, Water Resour. Res. 16, 1016 (1980).

[44] S. K. Matthäi and M. Belayneh, Geophys. Res. Lett. 31, L07602 (2004).

[45] N. W. A. Odling, S. C. Elphick, P. Meredith, I. Main, and B. T. Ngwenya, Earth Planet. Sci. Lett. 260, 407 (2007).

[46] E. Cey, D. Rudolph, and R. Therrien, Water Resour. Res. 42, W09413 (2006). 